\title{
Co-teaching y diversidad funcional cognitiva: una experiencia de sistematización con las TIC en educación superior
}

\author{
Pérez - Pérez, Itahisa \\ Universidad Pablo de Olavide, Sevilla, España \\ iperper@upo.es
}

Rodríguez - Izquierdo, Rosa M.

Universidad Pablo de Olavide, Sevilla, España

rmrodizq@upo.es

\section{Resumen}

En esta comunicación presentamos una experiencia de sistematización en el uso de las Tecnologías de la Información y la Comunicación (TIC), desde la reflexividad crítica, con personas con diversidad funcional cognitiva en el ámbito universitario. Entendiendo la Universidad como agente clave y decisivo en la inclusión social, ya que permite compartir espacios de formación, socialización, aprendizaje y crecimiento entre el alumnado con y sin diversidad funcional cognitiva, desarrollamos el "co-teaching» como modelo de compromiso docente y trabajo colaborativo en los equipos de aula con un grupo de estudiantes con diversidad funcional cognitiva. El «co-teaching» consiste en la gestión del trabajo de aula por parte de diversos docentes para la enseñanza y aprendizaje de todo el alumnado, con objeto de alcanzar una mayor eficacia. Este modelo se desarrolla en la asignatura TIC, en la cual el alumnado se aproximará al uso y manejo de herramientas tecnológicas para integrarse en el mundo sociolaboral, adoptando actitudes de respeto, participación, esfuerzo y colaboración que posibiliten la creación de producciones audiovisuales colectivas. La metodología implementada es participativa e inclusiva, basada en un planteamiento didáctico capaz de responder a las necesidades formativas de todo el alumnado, con actividades y materiales accesibles. Con el uso de los Núcleos de Aprendizajes Prioritarios (NAP), a través de la voz, el cuerpo, el juego dramático, los cuentos y las producciones visuales, integramos lo cognitivo, lo emocional y lo conductual, propiciando la comunicación y expresión a través de diferentes lenguajes verbales y noverbales.

\section{Abstract}

This paper presents a systematized experience in the use of Information and Communication Technologies (ICT), from critical reflection, with university students with cognitive functional diversity. Understanding the University as a key and decisive agent in social inclusion, since it allows sharing spaces for training, socialization, learning and growth between students with cognitive functional diversity and other students, "co-teaching" was developed as a model of teaching commitment and collaborative work with a group of students with cognitive functional diversity.The "co-teaching" consists in the management of classroom work by various teachers for the teaching and learning of all students, in order to achieve greater efficiency. This model is developed in an ICT course, in which students learnt the use and management of technological tools that could be useful for their integration in the social and labour environment, adopting attitudes of respect, participation, effort and collaboration that enable the creation of collective audiovisual productions. The methodology implemented is participatory and inclusive, based on a didactic approach capable of responding to the training requirements of all students, with accessible activities and materials. With the use of the Core Learning Priorities (NAP), through the voice, the body, dramatic play, stories and visual productions, the cognitive, emotional and behavioral were integrated, promoting communication and expression through different verbal and non-verbal languages.

Palabras clave: co-enseñanza, diversidad funcional, Educación Superior, educación inclusiva, TIC.

Keywords: co-teaching, functional diversity, Higher education, inclusive education, ICT.

\section{INTRODUCCIÓN}

Cada vez es más frecuente el acceso de las personas con diversidad funcional al sistema educativo español, aunque al pasar de la Enseñanza de Secundaria Obligatoria al Bachillerato se produce el «efecto 
embudo», que aumenta considerablemente al continuar estudios de Formación Profesional o Universidad. En estas etapas las tasas de abandono escolar temprano se duplican, con respecto al resto de la población estudiantil. Además, y aunque de la Enseñanza Universitaria no hay datos estadísticos, la atención al alumnado con diversidad funcional se ha convertido en estándar de calidad en el marco del Espacio Europeo de Educación Superior (EEES). En este contexto surge el curso «Formación para el empleo y la vida autónoma y de las personas con discapacidad intelectual», Título Propio de la Universidad Pablo de Olavide (UPO, Sevilla), subvencionado por la Fundación ONCE y el Fondo Social Europeo.

En el marco de este curso universitario se desarrolla la asignatura «Seminario TIC», correspondiente al módulo primero «Formación en comunicación, habilidades sociales, emocionales, entrenamiento cognitivo», en el primer semestre del curso académico 2017/2018, con un total de $15 \mathrm{~h}$.

Desde la reflexividad pretendemos valorar las posibilidades que ofrecen las TIC (Tecnologías de la Información y la Comunicación) para el ámbito personal, profesional y social, del alumnado con diversidad funcional cognitiva, mediante un uso seguro, responsable y consciente. Para ello desarrollamos una metodología centrada en el estudiante, fomentando el aprendizaje constructivo y significativo a través del uso de dispositivos móviles y la producción multimedia con finalidad expresiva, comunicativa o ilustrativa.

Tras la revisión literaria realizada y la experiencia de innovación docente implementada, consideramos necesario fomentar el modelo de co-teaching en los entornos educativos ya que, como señala Arndt y Liles (2012), es necesario proporcionar al profesorado oportunidades de colaboración con otros docentes dentro del aula. De esta manera, fomentamos la coordinación docente (tanto vertical como horizontal), atendemos a una educación inclusiva, brindamos una educación personalizada y contribuimos a crear e institucionalizar estructuras colaborativas, basadas en la igualdad de oportunidades, la equidad, el compromiso con el aprendizaje y el fortalecimiento de los contenidos y competencias necesarias para la educación integral, entre otros aspectos claves (Cobb Morocco \& Mata Aguilar, 2009).

\section{DIVERSIDAD FUNCIONAL COGNITIVA Y LAS TIC}

Tradicionalmente nuestra sociedad ha considerado a una persona alfabetizada aquella que sabe leer y escribir. Sin embargo, no basta sólo con estas funciones necesarias, ya que la persona se encontrará aislada de un gran flujo de información y comunicación que caracteriza a la sociedad actual. Como señala Rodríguez de las Heras (2006, en Luque Parra y Luque-Rojas, 2012: 28) nuestra sociedad «ha pasado de una sociedad de la información a una sociedad del conocimiento, lo cual implica una transformación social global».

Alfabetizar mediáticamente, según la UNESCO (2011a), significa conseguir una ciudadanía informada, entendiendo ésta como aquella que conoce y maneja las fuentes de forma crítica, adaptada a sus necesidades de aprendizaje, que comunica y expresa su posición de forma fundamentada. Esta alfabetización es importante que llegue a todos los colectivos, sobre todo aquellos en riesgo de exclusión social, como pueden ser las personas con diversidad funcional. Como señal an Luque Parra y Luque-Rojas (2012:29), este colectivo requiere "un mayor esfuerzo de superación de sus propias limitaciones para evitar el riesgo de exclusión... y más aún en las personas con discapacidad intelectual».

El uso de las TIC aporta grandes beneficios para el aprendizaje, la inclusión y las personas con diversidad funcional, como por ejemplo (De Miguel García, 2014: 10 y 11):

- Aumenta la capacidad de almacenamiento y de procesamiento de la información.

- Mejora la memoria semántica.

- Centra la atención.

- Posibilita una mejor comprensión de lo abstracto.

- Mejora la generalización y el mantenimiento del aprendizaje.

- Fomenta la iniciativa y la constancia en las tareas.

- Favorece la reflexión.

- Optimiza la organización temporal.

- Potencia la adquisición de aprendizajes como la memoria visual.

- Mejora la adquisición de conocimientos a través de varios canales sensoriales. 
- Motiva el aprendizaje a través de actividades educativas, lúdicas y de respuesta inmediata.

- Etc.

Nuestra experiencia docente aproxima el uso y manejo de herramientas tecnológicas al alumnado con diversidad funcional cognitiva, para integrarse en el mundo sociolaboral, adoptando actitudes de respeto, participación, esfuerzo y colaboración, que posibiliten la creación de producciones audiovisuales colectivas.

\section{CO-TEACHING: MODELO DE COMPROMISO DOCENTE}

El «co-teaching» ha sido nuestro modelo pedagógico a la hora de diseñar, implementar y evaluar esta experiencia docente. Entendido, no como una mera colaboración sino como un modelo de compromiso docente y trabajo colaborativo en los equipos de aula, centrado en la pedagogía de Freinet, donde prevalece la horizontalidad en las relaciones, la participación activa de sus miembros y la cooperación desde su triple dimensión: cooperación entre el propio alumnado, entre las docentes y el alumnado y entre las docentes (Freinet,2004).

Se trata de distribuir los objetivos de aprendizaje desde diversas maneras de enfocar la tarea, las estrategias, los recursos y las metodologías de aula (Pagés, 2017). Este paradigma pedagógico se centra en coordinar el trabajo para lograr metas comunes, compartiendo un sistema de creencias que respeta el posicionamiento y la mirada de cada docente, así como en utilizar un liderazgo compartido y distributivo de funciones (Rodríguez, 2015).

Con el uso de los Núcleos de Aprendizajes Prioritarios (NAP), a través de la voz, el cuerpo, el juego dramáti$\mathrm{co}$, los cuentos y las producciones visuales, integramos lo cognitivo, lo emocional y lo conductual, propiciando la comunicación y expresión a través de diferentes lenguajes verbales y no verbales, a través de las TIC.

\section{METODOLOGÍA}

La metodología docente implementada se caracteriza por ser activa-participativa, donde el alumnado sea el protagonista de su proceso de enseñanza aprendizaje; inclusiva, atendiendo de manera personalizada a las necesidades de cada estudiante, con actividades y materiales accesibles.

Las propuestas didácticas invitan a la progresión de los objetivos y contenidos pedagógicos, regidas por un orden de complejidad en el uso y manejo de las TIC. Además, este modelo pedagógico propicia la comunicación y expresión a través de los diferentes lenguajes verbales y no verbales, adquiriendo así mayor seguridad en los recursos propios y en las relaciones con los demás. Todo ello proyectado hacia la creación de un material de divulgación y sensibilización, donde el alumnado incorpora los trabajos realizados durante las diferentes sesiones, el material multimedia elaborado y muestra las competencias alcanzadas durante toda la formación.

Los objetivos que perseguimos en esta experiencia docente fueron:

- Objetivo 1. Adquirir habilidades de manejo de tecnologías de la información y la comunicación,que les permitan resolver con eficacia tareas relacionadas con su trabajo.

- Objetivo 2. Manejar herramientas tecnológicas necesarias para integrarse en las redes sociales, aportando sus competencias al crecimiento de las mismas y adoptando las actitudes de respeto, participación, esfuerzo y colaboración que posibiliten la creación de producciones colectivas.

- Objetivo3. Utilizar dispositivos para capturar y digitalizar imágenes, textos y sonidos para crear pequeñas producciones multimedia con finalidad expresiva, comunicativa o ilustrativa.

- Objetivo 4. Valorar las posibilidades que ofrecen las TIC para el ámbito personal, profesional y social, y en el ámbito del conocimiento.

Participaron en esta experiencia docente 14 de las 15 personas matriculados en la asignatura (véase Figura 1), ya que es importante la ratio del grupo para conseguir esa personalización e individualización de la formación. 
Figura 1. Los protagonistas.

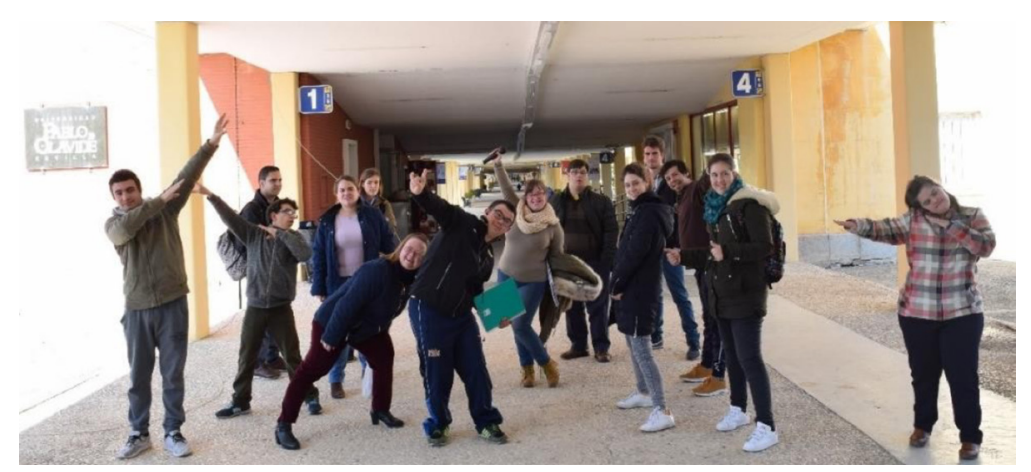

Fuente: elaboración propia.

De este grupo de 14 estudiantes, se subdividieron en tres equipos de trabajo, donde todas las personas ejecutaban las mismas tareas con el propósito de que aprendiesen haciendo. Las tareas a realizar eran: diseño del vídeo, elaboración de guión, preparación de la escenificación, elección de la música, captación de fotografías y grabación. Los equipos de trabajo se denominaron: los Tiburones en la UPO, los Fantásticos de la UPO y los Universitarios.

Con respecto a la temporalización, la experiencia se implementó desde mediados del mes de noviembrede 2017 hasta final de enero de 2018, con un total de 15h, distribuidas en las sesiones que presentamos en la Figura2:

Figura 2.Cronograma.

\begin{tabular}{|c|c|c|c|}
\hline SESIÓN Y FECHA & HORARIO & N $^{\circ}$ HORAS & TEMA \\
\hline \multirow{2}{*}{ y y $2(16 / 11 / 17)$} & $10^{\prime} 00-11^{\prime} 30$ & $1^{\prime} 5$ & Bloque I \\
& $12^{\prime} 00-13^{\prime} 30$ & $1^{\prime} 5$ & Bloque I y II \\
\hline \multirow{2}{*}{3 y $4(27 / 11 / 17)$} & $10^{\prime} 00-11^{\prime} 30$ & $1^{\prime} 5$ & Bloque II \\
& $12^{\prime} 00-13^{\prime} 30$ & $1^{\prime} 5$ & Bloque II y III \\
\hline \multirow{2}{*}{5 y $6(30 / 11 / 17)$} & $10^{\prime} 00-11^{\prime} 30$ & $1^{\prime} 5$ & \\
\hline \multirow{2}{*}{7 y $8(01 / 12 / 17)$} & $12^{\prime} 00-13^{\prime} 00$ & 1 & Bloque III \\
\hline \multirow{2}{*}{ Sesión 9 y 10 (23/01/18) } & $10^{\prime} 00-11^{\prime} 30$ & $1^{\prime} 5$ & \\
\hline TOTAL & $12^{\prime} 00-13^{\prime} 30$ & $1^{\prime} 5$ & \\
\hline
\end{tabular}

Fuente: elaboración propia.

\section{DISCUSIÓN Y CONCLUSIONES}

Para corroborar la consecución de los objetivos hemos desarrollado una evaluación continua ya que, mediante la observación, acompañamiento y tutorización llevada a cabo durante las sesiones de trabajo, se iba verificando la adquisición de conocimiento y manejo de las TIC. Además, se ha ido adaptando el contenido y la metodología a medida que íbamos desarrollando las sesiones, de manera que estuviese la formación personalizada, a cada necesidad concreta, respondiendo así tanto a las necesidades advertidas (aquellas que el alumnado reconocía y expresaba), las latentes (aquellas que existen pero que el alumnado no reconoce o exterioriza) y «otras necesidades de las cuales la población no es consciente, pero que es preciso despertar por ser inseparables de la dignidad y del bienestar humanos» (Nogueiras Macareñas, 1996:67). De esta manera, hemos sido testigos de un crecimiento no sólo profesional, sino que también personal del alumnado. 
La creación del material audiovisual divulgativo elaborado por el alumnado ha facilitado la adquisición y aplicación de conocimientos, herramientas, estrategias, destrezas y habilidades necesarias en las TIC, y para su formación integral, a través de los NAP. Este material recoge sus experiencias tanto por la oportunidad que ha supuesto poder estudiar en la universidad, como la autonomía que conlleva (transporte desde sus casas hasta el recinto universitario, relaciones interpersonales con personal de administración y servicio, alumnado de otros cursos, personal de cafetería, docentes, etc.) y los aprendizajes adquiridos. Además, comparten sus impresiones sobre la semana de inmersión comunitaria que vivieron en la residencia universitaria, ubicada en una Zona con Necesidades de Transformación Social (ZNTS) y donde pudieron visitar varios centros educativos, entidades sociales, etc.

El vídeo de sensibilización creado por el grupo de estudiantes se denomina «Pido la Palabra. Estudiantes con diversidad funcional cognitiva en la UPO», y se podrá visionar en el repositorio multimedia UpoTV de dicha universidad (https://upotv.upo.es/).

La evaluación general del alumnado con respecto a esta experiencia docente ha sido muy satisfactoria para todas las personas participantes, considerando la asignatura "Seminario TIC" como muy relevante. Además, han valorado muy positivamente a las docentes, así como la metodología implementada, las relaciones interpersonales que se han producido dentro y fuera del aula, los contenidos trabajados y todo el proceso de diseño, elaboración, escenificación y grabación del material.

En cuanto al modelo pedagógico de co-teaching, presentamos a continuación un DAFO con el análisis crítico de las docentes:

\section{Figura 3. DAFO Co-teaching}

\begin{tabular}{|c|c|}
\hline Debilidades & Amenazas \\
\hline $\begin{array}{l}\text { La falta de tiempo en la asignatura dificulta realizar } \\
\text { un proceso con cada estudiante para comprobar qué } \\
\text { funciona y que no. } \\
\text { La falta de tiempo entre las docentes, y con el equipo } \\
\text { de todo el título, dificultad el poder integrar los } \\
\text { aspectos que se necesitan modificar. }\end{array}$ & $\begin{array}{c}\text { El riesgo de que la organización del módulo varíe } \\
\text { para ediciones futuras y haya que comenzar a } \\
\text { repensar de nuevo la asignatura. }\end{array}$ \\
\hline Fortalezas & Oportunidades \\
\hline $\begin{array}{l}\text { Clarificación y reprogramación de los roles y } \\
\text { responsabilidades entre las docentes. } \\
\text { Apertura para compartir conocimientos y percepciones. } \\
\text { Respeto, responsabilidad y compromiso compartido. } \\
\text { Estrategias de organización y manejo efectivo del aula. }\end{array}$ & $\begin{array}{l}\text { Seguir mejorando profesionalmente a través de } \\
\text { la reflexión compartida de la experiencia con un } \\
\text { colectivo con el que no estamos habituadas a } \\
\text { trabajar y que de por si es muy heterogéneo. } \\
\text { Mayor número de horas en la asignatura para } \\
\text { adaptarnos mejor a los diferentes } \\
\text { ritmos de aprendizaje. }\end{array}$ \\
\hline
\end{tabular}

Fuente: elaboración propia.

Las TIC por sí solas no garantizan la adquisición ni construcción del conocimiento, sino que «hay que proporcionar un entorno que facilite la interacción social, la correcta utilización de los medios y la experimentación. En este sentido, hay sugerencias metodológicas de tutorización, estrategias de dinamización, gestión del tiempo, trabajo colaborativo y propuestas de evaluación» que pueden facilitar esta integración de la tecnología y la alfabetización mediática enelaula (Tormo Calandín, Tejeda Adell, Romero-Gómez y Domingo Triado, 2014:31).

Tras todo lo expuesto, consideramos que las claves de éxito de este modelo pedagógico, basado en el co-teaching, han sido: el diseño y la planificación de la intervención, la adaptación de contenidos, junto con el material online adaptado, y la evaluación continua como proceso cíclico de análisis y reflexión sobre la adquisición de conocimientos, destrezas y habilidades, adaptándolas a las características de cada estudiante y sus diferentes ritmos.

La coordinación docente en co-teaching ha sido fundamental para transmitir un mismo mensaje, integrando dos modelos docentes diferentes, con las ventajas que aporta una doble mirada de la misma realidad, brindando una oportunidad para el desarrollo profesional docente. "Y es que la práctica de 
la pareja pedagógica no significa anular estilos docentes, si no aportar la esencia de lo que cada uno es aun acto de enseñanza aprendizaje en común, para transformarlo en algo distinto y mejor que lo que cada uno es por separado» (Cotrina García, García García y Caparrós Martín, 2017:61).

En definitiva, el uso y manejo de las TIC es «una herramienta fundamental para introducir nuevas miradas en los procesos educativos, así como para repensar los modelos pedagógicos empleados en la enseñanza» (Prefasi, Magal, Garde y Giménez, 2010: 110). Y, como muestra gráfica del proceso de aprendizaje de lalumnado, así como de la creación del material audiovisual, finalizamos este trabajo con el eslogan de «Pido la palabra».

Figura 4. Eslogan de «Pido la palabra».

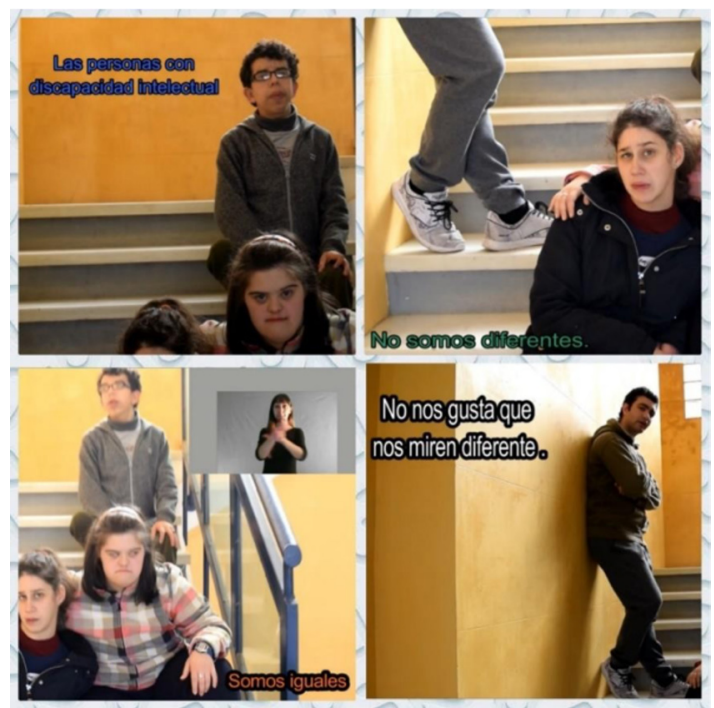

Fuente: elaboración propia.

\section{REFERENCIAS BIBLIOGRÁFICAS}

Arndt, K. \& Liles, J. (2012). Preservice Teachers' Perceptions of Coteaching: A Qualitative Study, Action in Teacher Education, 32:1, 15-25, DOI: 10.1080/01626620.2010.10463539.

CobbMorocco, C. \& Mata Aguilar, C. (2009). Coteaching for Content Understanding: A School wide Model, Journal of Educational and Psychological Consultation, 13:4, 315-347, DOI: 10.1207/S1532768XJEPC1304_04.

Cotrina García, M; García García, M. y Caparrós Martín, E.(2017). Ser dos en el aula: las parejas pedagógicas como estrategia de co-enseñanza inclusiva en una experiencia de formación inicial del profesorado de secundaria, en Aula Abierta, n. ${ }^{\circ} 46$, pp.57-64.

Freinet, E. (2004). Pedagogía Freinet. Los equipos pedagógicos como método. Alcalá de Guadaira: Editorial Trillas.

Luque Parra, D. J; Luque-Rojas, M. ${ }^{\text {. }}$ J. (2012). Aspectos psicoeducativos en las relaciones de las TIC y la discapacidad intelectual, en Revista Intercontinental de Psicología y Educación, vol. 14, núm. 1, enero-junio, pp. 27-48. En http:// www. redalyc.org/articulo.oa?id=80224034003.

Nogueiras Macareñas, L. M. (1996). La práctica y la teoría del Desarrollo Comunitario. Descripción de un modelo. Madrid:Narcea.

Pagès, A. (2017). El 'co-teaching', una forma de educar con compromiso. En La Vanguardia, en http://www.lavanguardia. com/vida/20170413/421651069581/co-teaching-escuela-participacion-rendimiento-escolar.html.

Prefasi, S; Magal, T; Garde, F. y Giménez, J. L. (2010). Tecnologías de la Información y de la Comunicación orientadas a la educación de personas con discapacidad cognitiva, Revista Latinoamericana de Tecnología Educativa -RELATEC, 9 (2), pp. 107-123. En http://campusvirtual.unex.es/revistas/index.php?journal=relatec.

Rodríguez, F. (2015). La co-enseñanza, una estrategia para el mejoramiento educativo y la inclusión. En Revista Latinoamericana de Educación Inclusiva, vol. 8, n² 2, pp. 219-233.

Tormo Calandín, C; Tejeda Adell, M; Romero - Gómez, B. y Domingo Triado, V. (2014). Metodología docente cooperativa-colaborativa en el Grado de Medicina. En Therapeía, 6, pp.13-36.

UNESCO (2011a). Alfabetización Mediática e Informacional. Currículum para profesores. París: Organización de las Naciones Unidas para la Educación, la Ciencia y la Cultura. En http://unesdoc.unesco.org/images/0021/002160/216099s. pdf, pp. 16-39. 